\title{
Khan Academy como herramienta en el aprendizaje de las matemáticas y la programación de computadoras
}

\author{
Luis Adrián Lasso Cardona \\ Karen Natalia Conde Rodríguez ${ }^{2}$
}

Recibido: 03-05-2020

Aceptado: 06-05-2020

\section{Resumen}

La investigación identificó la percepción acerca de la relación entre las matemáticas y las ciencias de la computación, y de cómo Khan Academy ayuda al estudio en estas áreas. La relación entre matemáticas y computación, fundamental en el desarrollo de nuevas tecnologías, debe ser impartida con enfoque constructivista y conectivista, y apoyada en TIC, buscando que el estudiante desarrolle desde muy temprana edad sus capacidades y el interés hacia carreras STEM. Se concluye que el uso de Khan Academy fue muy positivo: los resultados muestran que para el $83 \%$ de los estudiantes la herramienta logró resolver gran parte de sus interrogantes. Así mismo, para el $67 \%$ los temas tratados en los cursos seleccionados les permitieron aprender o reforzar sus conocimientos, y para el $82 \%$ el uso de la plataforma obtuvo una calificación entre 4 y 5 .

\footnotetext{
1. Ingeniero de sistemas, Universidad del Valle, Colombia. M.Sc. en Gestión de la Tecnología Educativa, Universidad de Santander, Colombia. Profesor asistente, Universidad del Valle. SIEL (Investigación y Desarrollo en Sistemas de Información y Electrónica), Univalle, Sede Buga, Colombia.

ORCID: https://orcid.org/0000-0002-3354-1554

Correo electrónico: luis.lasso@correounivalle.edu.co

Google académico: https://scholar.google.com/citations?user=YdXtijOAAAAJ\&hl=es\&oi=ao

CvLac: https://scienti.minciencias.gov.co/cvlac/visualizador/generarCurriculoCv.do?.cod_ $\mathrm{rh}=0000156514$

2. Estudiante de la Facultad de Ciencias de la Computación, Universidad del Valle, Sede Palmira, Colombia.

ORCID: https://orcid.org/0000-0003-3938-2248

Correo electrónico: karen.conde@correounivalle.edu.co

Google académico: https://scholar.google.com/citations?user=Q0nuV9cAAAAJ\&hl=es\&authuser=1

CvLac: https://scienti.minciencias.gov.co/cvlac/visualizador/generarCurriculoCv.do?.cod_ rh=0001770914
} 
Palabras clave: TIC, RAE, STEM, matemáticas, computación, programación de computadoras, constructivismo, conectivismo.

\title{
Khan Academy as a tool in learning mathematics and computer programming
}

\begin{abstract}
The research identified the perception of the relationship between mathematics and computer science, and how the Khan Academy helps study in these areas. The mathematical and computing relationship, fundamental in the development of new technologies, must be taught with a constructivist and connectivist approach, and supported by ICT, seeking for the student to develop from an early age their abilities and interest in STEM careers. It is concluded that the use of Khan Academy was very positive, showing that for $83 \%$ of the students the tool managed to solve many of their questions. Likewise, for $67 \%$, the topics covered in the selected courses allowed them to learn or reinforce their knowledge, and for $82 \%$, the platform obtained a score between 4 and 5 points of acceptance in the use of the platform.
\end{abstract}

Keywords: ICT, RAE, STEM, mathematics, computing, computer programming, constructivism, connectivism.

\section{Introducción}

La humanidad está viviendo avances tecnológicos que obligan a reformar las estructuras de sectores como la educación, donde los modelos de aprendizaje flexibles basados en nuevas tecnologías buscan ser una alternativa para una educación más cercana, abrien- 
do nuevas oportunidades de capacitación (OECD, 2016) y reduciendo la brecha digital entre los alumnos (Rumiche, Matas y Ríos, 2020). En este sentido, la Estrategia Europea 2020 incentiva la creación de entornos de aprendizaje interactivos con el uso de tecnologías de la información y las comunicaciones (TIC) en las instituciones de educación superior (Ortiz, Santos y Marín, 2020). En Colombia, el Plan Nacional Decenal de Educación 2016-2026 del Ministerio de Educación (MEN) estableció la necesidad de impulsar el uso de tecnologías para la enseñanza, la investigación y la innovación, y lograr una educación de calidad (MEN, 2019).

Actualmente, la alfabetización digital está enfocada en formar usuarios con habilidades para interactuar con la tecnología principalmente como mecanismo de comunicación y aprendizaje. No obstante, debido a la carencia mundial de profesionales informáticos (Romero, Cano, Charry y Pardo, 2019; Rubio, 24 de septiembre de 2019; La República, 28 de agosto de 2019) ha surgido un creciente interés por parte de las economías en ampliar la formación tecnológica de los jóvenes en cuanto al desarrollo de nuevas tecnologías con algoritmos computacionales capaces de procesar los datos generados desde múltiples fuentes y dar respuesta a las necesidades de la sociedad (Jara y Hepp, 2016). En Colombia, el Plan Estratégico Institucional 2019-2022 del MEN, en concordancia con los Objetivos de Desarrollo Sostenible (ODS) de la Organización de las Naciones Unidas para la Educación, la Ciencia y la Cultura (Unesco), advirtió la necesidad de aumentar la oferta de estudios en ingeniería y tecnologías de la información como factores de progreso y mejoramiento de la calidad de vida de manera sostenible (MEN, 2019).

La revolución tecnológica demanda profesionales cualificados en diversas ciencias como la matemática, con capacidad de adaptación y respuesta a las demandas del entorno. Es así como el desarrollo de estas competencias está estrechamente relacionado con la creación, implementación y uso de nuevas tecnologías (García, Gómez, Solano y Fernández, 2020).

A pesar de que la matemática es un área fundamental para el desarrollo intelectual, lógico y crítico, aplicable en diferentes con- 
textos de la vida (Minte, Sepúlveda, Díaz y Payahuala, 2020), y de que pertenece a los currículos escolares de educación básica y universitaria, su proceso de enseñanza-aprendizaje aún presenta dificultades (Alvites, 2017) relacionadas con factores sociales (García y Romero, 2018; Palmas, 2018), económicos, pedagógicos y epistemológicos (Vesga y Losada, 2018; Cruz Portillo, Tchoshanov, Flores y Ramírez, 2019), y con condiciones especiales (Valbuena, Padilla y Rodríguez, 2018), el tipo de educación (oficial o privada) y factores psicológicos. Por ejemplo, la ansiedad en el alumno le hace creer que es incapaz de afrontar la materia, por lo cual toma una actitud indiferente que se refleja en calificaciones bajas (Jiménez et al., 2020). Así mismo, la ansiedad hacia las matemáticas es un factor no intelectual que afecta a estudiantes con alto rendimiento en otras materias, por lo que muchos se abstienen de cursar estudios profesionales que involucren esta asignatura, lo que puede dar como resultado una pérdida de talentos en las áreas científicas (López, Mato y Chao, 2020).

Para abordar esta problemática, la orientación del MEN es potenciar las habilidades en los estudiantes mediante la interacción del aprendizaje matemático de experiencias reales, que incluyan la comunicación, la argumentación y la participación del estudiante en el proceso (Valbuena, Muñiz y Berrío, 2020), similar al modelo EEES (Espacio Europeo de Educación Superior) en Europa (Lluch y Portillo, 2018), haciendo que el aprendizaje no sea regido por definiciones y procesos mecánicos, sino que, por el contrario, sirva de fórmula para encontrar una relación de aplicación entre lo aprendido y el plan de estudios de otras materias (Olarte, 2020), permitiendo que el profesor reconozca patrones de pensamiento en los estudiantes, mejore la comunicación y aplique recursos tecnológicos que reformen el proceso de enseñanza (Solar, 2018).

Al observar los currículos profesionales de las carreras asociadas a las ingenierías, se puede notar que el cálculo mental y el razonamiento matemático son habilidades necesarias que deben ser desarrolladas y entrenadas desde muy temprana edad, las cuales dan un nivel superior de pensamiento (Gürbüz y Erdem, 2016). En el caso de las ciencias de la computación, ambas ha- 
bilidades están presentes, pues se utilizan para el diseño de algoritmos computacionales que resuelvan problemas en sistemas simples y complejos, y que sin la ayuda de la informática serían prácticamente irresolubles (Gasca y Machuca, 2017). Básicamente, la programación de computadoras es el uso de lenguajes de programación que, apoyados sobre una base lógica-matemática sólida, ayudan a diseñar, implementar y probar el software (Díaz, Fierro y Muñoz, 2018). Por ejemplo, para el desarrollo de videojuegos es necesario usar álgebra lineal, geometría, vectores y transformaciones para lograr el movimiento de objetos y personajes, y aplicar la teoría de grafos para construir las estrategias del juego.

Para la Organización para la Cooperación y el Desarrollo Económicos $(\mathrm{OCDE})$, existen seis grandes áreas científicas, entre las que se encuentran la ingeniería y la tecnología, que están relacionadas con las matemáticas, la estadística, la geometría, la física y las ciencias de la computación. De igual forma, el MEN considera ocho áreas del conocimiento, entre ellas la ingeniería y las matemáticas, que comprenden núcleos básicos como la telemática, sistemas y telecomunicaciones, entre otros (Cárdenas-Guevara, 2015), y dentro de las cuales las matemáticas discretas, el cálculo, los métodos numéricos, la estadística y la programación lineal juegan un papel importante en el profesional informático (LopezGonzález, 2017).

Muchas investigaciones demuestran la importancia en la relación entre matemáticas y ciencias de la computación. En el campo gerencial, Arba (2019) utiliza una simulación de Monte Carlo para mejorar el análisis de riesgo y costos utilizado en agencias de carreteras en Estados Unidos. Yusuf, Lado y Ali (2020) aplican modelos matemáticos para mejorar el rendimiento de un sistema de red de comunicación. Helizar, Isnanto y Nurhayati (2018) proponen el modelo SEM (Structural Equation Modelling) para la implementación de sistemas ERP (Enterprise Resource Planning). Shen (2020) estudia las redes de cadenas de suministro con variables inciertas, y Muhammad, Garba Oye y Wajiga (2019) usan la teoría de conjuntos difusos para desarrollar un sistema experto. 
En seguridad y criptografía, Bouroubi, Charchali y Benyahia (2019) presentan un nuevo protocolo criptográfico para compartir claves en canales inseguros. James, Thumbur y Vasudeva (2020) desarrollaron un esquema de proxy más seguro y eficiente computacionalmente. Ashir, Akdemir y Ahmed (2018) proponen un sistema para reconocimiento facial usando la distancia de Manhattan. Cariow, Cariowa y Majorkowska (2020) diseñaron un algoritmo de rotación 3D basada en Quaternion para visión artificial; y Byrd (2018) expone la importancia de diseñar algoritmos más complejos con simulaciones Monte Carlo, cadenas de Markov, teoría de juegos y estadísticas de Poisson para modelar riesgos de internet.

En análisis de datos, Ghaleb, Taha, Hazman, Abd ElLatif y Abbass (2020); Saouabi y Ezzati (2020); Wenqi y Jian (2019), Hasselman y Bosman (2020); Salas, Poveda, Mesa y Marwan (2020), y Segarra, Pérez, Moya, Ayuso y Beltrán (2020) utilizan algoritmos matemáticos y modelos estadísticos para visualizar grandes cantidades de datos y encontrar patrones de comportamiento social y climático. En medicina, Joshi, Sharma y Prajapati (2020), y Badmus, Alexander y Florence (2020) usaron estadística para crear un modelo computacional que analiza el virus del Ébola y otras enfermedades. Emeda, Mwalili y Otieno (2020), y Elaiw y Alshaikh (2020) propusieron un modelo de ecuación diferencial y modelos dinámicos, respectivamente, para estudiar el VIH y el sida. Yuan, Liu, Huang y Hu (2020), y Ramírez, Aparicio y Gómez (2019) implementaron técnicas de aprendizaje automático para analizar imágenes de resonancia magnética e identificar cáncer. Zhao et al. (2020), Acharya, Oh, Hagiwara, Tan y Adeli (2018), Ullah, Hussain, Qazi y Aboalsamh (2018), Türk y Özerdem (2019) y Hussein, Palangi, Ward y Wang (2019) crearon modelos computacionales para la identificación automática de la epilepsia mediante redes neuronales.

En la industria, Alshammari y Rezgui (2020), Trejo, Fajardo y Gutiérrez (2020) y Popoli, Cristofolini y Sandrolini (2020) utilizaron grafos para optimizar tiempos en cruces de semáforos y la interferencia electromagnética en líneas eléctricas. 
Mundialmente, muchos países están reformando sus currículos para incluir la enseñanza de la programación y el pensamiento computacional en varios niveles escolares. En 2018 la Comisión Europea dio a conocer el Plan de Acción de Educación Digital, donde esto se establecía como uno de los principales objetivos. En Estados Unidos, el Gobierno lanzo el proyecto "Computer Science for All", encaminado a que los estudiantes, desde la educación infantil, aprendan ciencias de la computación. Iniciativas similares se efectúan en Singapur, Nigeria y Australia (MEFP, 2018). En Colombia, una propuesta impulsada por el MEN y el Ministerio de las TIC tiene como estrategias el financiamiento de estudios y proyectos de investigación, la definición de un modelo para la industria de software nacional que permita aumentar el número de empresas TIC, el ajuste de currículos en la educación básica y superior, el impulso de la educación virtual y la creación de cursos MOOC (massive open online course) en temas de TIC para la formación de la ciudadanía (Colciencias, 2018).

Pese al aumento de aplicaciones de software y plataformas virtuales educativas, el aprendizaje en todas las áreas de la matemática básicamente tiene dos métodos. Uno enfocado a la enseñanza de procedimientos algorítmicos para dar solución a problemas tradicionales, y el segundo enfocado en demostraciones y formulismo matemático que no aporta en la construcción de conocimientos (Prieto y Cabrera, 2020; Vargas, Escalante y Carmona, 2017; Fonseca y Alfaro, 2018). En este aspecto, metodologías más dinámicas como STEM (Science, Technology, Engineering and Mathematics) son usadas dentro de los pénsums tradicionales, aumentando en 1,5 veces la probabilidad de éxito en cursos de ingeniería, matemática y ciencias (Reyes, Saavedra y Aguayo, 2020). Las investigaciones de Aranda y Callejo (2017), Ortiz y Padilla (2017), Sobalvarro y Camacho (2018), Briz y Serrano (2018), Segade y Naya (2018), García (2018), Hernández (2018) y Vilca (2019), realizadas en diferentes niveles educativos en el uso de Geogebra, lenguajes de programación R y Scratch, y la modernidad en los currículos, muestran la importancia de incluir la tecnología en áreas STEM. 
La propuesta STEM nació en Estados Unidos en 2011 y desde entonces muchos países la han incluido en sus políticas educativas, con un enfoque práctico que relaciona los contenidos curriculares con la realidad (Fuentes y González, 2019), para que con ayuda de la ciencia, la tecnología, la ingeniería y las matemáticas se pongan en práctica los conocimientos para resolver problemas en diferentes contextos sociales (Domínguez, Oliveros, Coronado y Valdez, 2019). El aprendizaje fuera del aula de las disciplinas STEM comúnmente pasa por la utilización de Recursos Educativos Abiertos (REA), que con ayuda de TIC son un medio de consulta abierto, ampliamente adoptado por muchos usuarios (Unesco, 2015). En matemáticas, calculadoras digitales como Wiris, MathPapa y Geogebra cambiaron la forma de aplicar la geometría y el álgebra. En ciencias, los laboratorios virtuales de física y química como PhET, Educaplus.org y ChemvLab permiten de forma divertida y segura realizar simulaciones. Así mismo, el aprendizaje de la programación de computadoras mediante los Laboratorios Virtuales de Programación (LVP) está aumentando en varios niveles educativos, más cuando la computación se asocia al lenguaje matemático en conceptos como variables, vectores y matrices, sistemas de coordenadas, y operadores lógicos, relacionales y aritméticos, indispensables para la construcción de algoritmos computacionales (López, Couso y Simarro, 2020), sumado a que un LVP reduce los gastos de equipamiento de un laboratorio real para el desarrollo de software, bases de datos, robótica y electrónica (Zaldívar, 2019).

Kahn Academy es uno de los RAE más populares a nivel mundial. Con más de 12 millones de usuarios al mes, presenta una metodología conectivista, donde los alumnos son premiados con puntos e insignias que representan el desempeño en cada tema, dando una motivación al proceso de aprendizaje (Kelly y Rutherford, 2017). De igual modo, los recursos disponibles en los cursos son mostrados según el nivel de conocimiento del participante, facilitando el autoaprendizaje y la colaboración (Rueda y Rey, 2018). Además, la plataforma permite analizar los resultados de las evaluaciones y compararlos con los resultados de los métodos habituales (Jara, Cancino y Casillas, 2019). Estudios realizados por Gray y Lindstrøm (2019), Zengin (2017), Dove y Dove (2017), Tenório, Lopes, Góis 
y Junior (2018), Rao, Hilton III y Harper, (2017) y Tapia (2019) argumentan el éxito en el uso de Kahn Academy como herramienta pedagógica dentro y fuera del aula de clase. Actualmente la plataforma ofrece cursos en diferentes áreas del conocimiento y nivel educativo. En matemáticas: aritmética, preálgebra, cálculo, probabilidad y estadística, ecuaciones diferenciales, geometría y álgebra lineal. En computación: programación de computadoras, ciencias de la computación, bases de datos y animación digital.

En este contexto, la presente investigación tiene dos objetivos: 1) conocer cuál es la percepción acerca de la relación que existe entre las matemáticas y las ciencias de la computación, y 2) descubrir el nivel de uso y aceptación del RAE Khan Academy como herramienta de aprendizaje en ambas áreas.

\section{Metodología}

Para abordar los temas de investigación, primero se efectuó una revisión documental en fuentes primarias que sentaron las bases teóricas de los temas a tratar, presentando definiciones y ejemplos que permitieron dar un contexto científico y práctico. Esta exploración inicial permitió identificar a Kahn Academy como el RAE más apropiado para la investigación, según las características ofrecidas y áreas de estudio. Se utilizó una metodología cuantitativa, orientada a recolectar datos que posteriormente son medidos y analizados para obtener conclusiones acerca del tema investigado (Cadena et al., 2017). También se empleó un enfoque descriptivo, con el propósito de reseñar la situación en cuanto al uso del RAE seleccionado por parte de los alumnos y la influencia en sus procesos de aprendizaje.

La recolección de datos se realizó por medio de una encuesta aplicada a una población de 132 estudiantes de diferentes semestres de las sedes Buga, Palmira y Tuluá de la Escuela de Ingeniería de Sistemas y Computación de la Universidad del Valle (Colombia). 


\section{Resultados}

Para realizar la figura 1, que representa el número de estudiantes con dificultades o deficiencias en las áreas de matemáticas y programación de computadoras, los investigadores crearon tres grupos de estudiantes categorizados por semestre. El primer grupo se denomina "Ciclo de fundamentación" e incluye a estudiantes de 1.er a 3.er semestre, que cursan las asignaturas: Cálculo I, II y III, Matemáticas Discretas I y II, Álgebra Lineal, Física I, Fundamentos de Programación, Programación Orientada a Objetos y Programación Interactiva. El segundo grupo se denomina "Ciclo de profundización" e incluye a estudiantes de $4 .^{\circ}$ a $7 .^{\circ}$ semestre, que cursan: Ecuaciones Diferenciales, Física II, Métodos Numéricos, Probabilidad y Estadística, Lenguajes de Programación, Desarrollo de Software I y II, Inteligencia Artificial y Simulación. Y el tercer grupo se denomina "Ciclo de profesionalización" e incluye a estudiantes de 8. ${ }^{\circ}$ a $10 .^{\circ}$ semestre, que cursan: Complejidad y Optimización, Aplicaciones en el Web y Sistemas de Información.

Figura 1. Dificultades en el aprendizaje de la matemática y la programación.

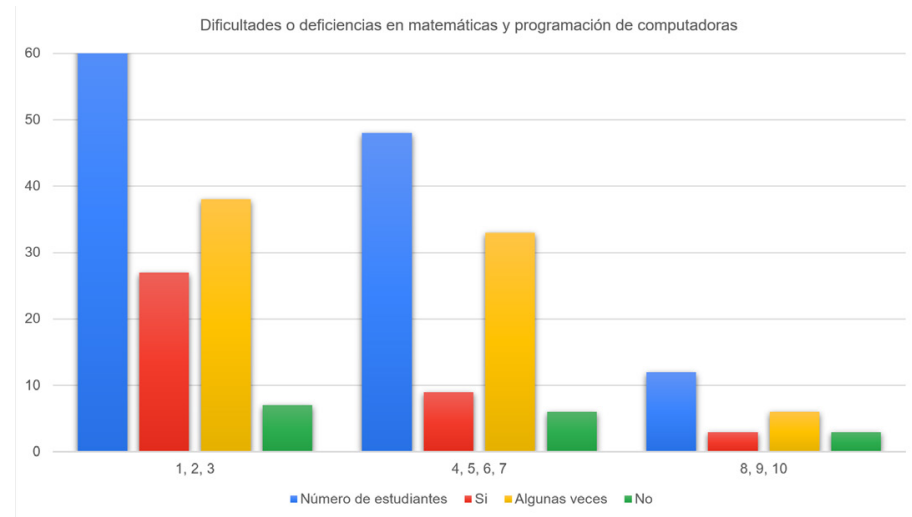

Fuente: elaboración propia

La estadística muestra que las mayores dificultades se presentan en los primeros semestres, donde un $90 \%$ de los estudiantes del Ciclo de fundamentación respondieron "sí" o "algunas veces", 
lo cual puede ser reflejo del escaso nivel educativo en las escuelas de secundaria, sobre todo en el área de matemáticas, uno de los factores más comunes en la deserción universitaria.

Por otra parte, antes de aplicar la encuesta se solicitó a los estudiantes que seleccionaran entre matemáticas y programación como áreas de estudio con Khan Academy, ya que era de interés conocer la relación que pudiera existir entre ambas. Los resultados se muestran en la figura 2. Se aprecia que en los tres grupos la computación, con un $43 \%$, fue el área más seleccionada, resultado que es normal y esperado según el perfil del programa académico. Después matemáticas, con $32 \%$ y ambas, con $26 \%$. Es de resaltar que nuevamente los estudiantes del "Ciclo de fundamentación" fueron los que mayormente seleccionaron el área de matemáticas, siendo Cálculo, Álgebra y Trigonometría las de mayor selección con un $46 \%$, $25 \%$ y $16 \%$, respectivamente, lo cual es conforme con lo expresado en el análisis de la figura 1. En cuanto a las áreas de computación, las de mayor selección fueron la programación de computadoras con $50 \%$, bases de datos con $34 \%$ y ciencias de la computación con un $32 \%$, asignaturas muy relacionadas con el proceso de ingeniería de software.

Figura 2. Áreas de estudio seleccionas con Khan Academy.

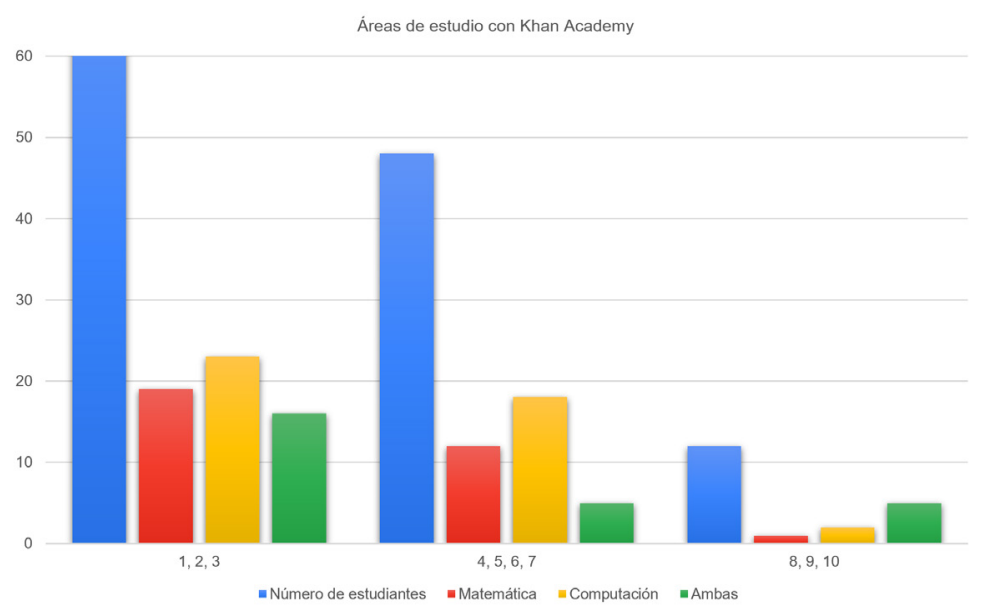

Fuente: elaboración propia 
Finalmente, los estudiantes, después de interactuar con la plataforma, dieron una calificación en una escala de 1 (el más bajo) a 5 (el más alto) al uso de la plataforma Khan Academy, y si cumplió con las expectativas en cuanto a la pertinencia y calidad de los temas tratados en cada curso, la flexibilidad, dinamismo y metodología. En la figura 3 se aprecia que la plataforma obtuvo muy buenos resultados en las tres categorías, arrojando un $82 \%$ entre la calificación de 4 y 5 , demostrando que la escogencia de Khan Academy fue acertada.

Figura 3. Escala de calificación (1-5) en el uso de la plataforma Khan Academy.

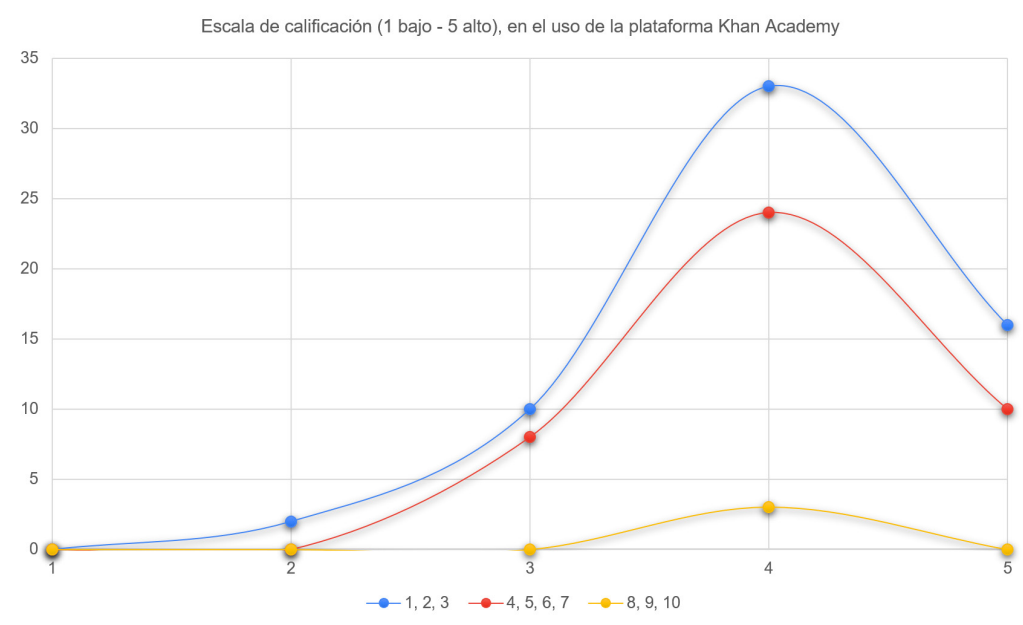

Fuente: elaboración propia

\section{Conclusiones}

El rápido avance en las tecnologías de comunicación y el aumento de nuevos lenguajes de programación han permitido la creación de herramientas novedosas como los RAE, que mejoran el proceso enseñanza y aprendizaje haciéndolo más flexible, dinámico y colaborativo, adaptándolo a las condiciones individuales de cada alumno (Revelo y Carrillo, 2018); es por ello que las escuelas e instituciones de educación superior a nivel mundial deben acogerlas, no solo 
como herramientas de apoyo en la labor docente, sino también incluirlas en los contenidos programáticos de todas las áreas, de tal forma que permita la construcción de proyectos integradores que den solución a problemas del aula, el entorno y el país.

En la actualidad, la web 3.0 ha posibilitado utilizar plataformas educativas como Khan Academy y MOOC que abren nuevas oportunidades de inclusión, disminución de la brecha digital y capacitación a la sociedad, al permitir de forma sencilla, fresca y divertida obtener y compartir conocimientos que en algunos casos son inexistentes en la educación tradicional, o que por factores económicos, sociales, geográficos o de condición física no sería posible lograr de otro modo. Este tipo de nuevas herramientas formativas son utilizadas por los estudiantes como estrategia para crear redes de comunidades alrededor del mundo, que se unen para intercambiar información, fomentar nuevas metodologías, incentivar la investigación y desarrollar conocimiento provechoso para el mejoramiento de la calidad de vida de sus comunidades (Grisales, 2018).

Por otra parte, el tema de la forma en que los estudiantes adquieren conocimiento está siendo muy investigado, y es importante en el rediseño de los planes curriculares y las estrategias de enseñanza, para impulsar el aprendizaje que responda a las necesidades didácticas de los actuales entornos, y actualice la manera en que las instituciones educativas, maestros y padres de familia afronten la labor de aprendizaje y seguimiento del desempeño académico (Coto, 2020).

Uno de los resultados más relevantes de la investigación es la identificación de que factores como la flexibilidad, el dinamismo, la colaboración, el conocimiento práctico y el aprendizaje a ritmo propio son fundamentales para los estudiantes de hoy en día, lo cual pone de manifiesto la necesidad de involucrar las TIC de manera más activa en los procesos de enseñanza-aprendizaje. Para los estudiantes los modelos tradicionales de enseñanza que fijan al maestro como el centro del conocimiento son cosa del pasado, y modelos con enfoques constructivistas que integren los saberes del pénsum de manera global son acogidos con mayor entusiasmo, 
y donde ellos son autónomos en regular y construir su aprendizaje de forma colaborativa (Alzahrani, 2017). De igual manera, la utilización de recursos tecnológicos como apoyo en los procesos de enseñanza por parte de los estudiantes es acogida de manera significativa; prueba de ello es que un $93 \%$ de los estudiantes encuestados considera su uso como completamente o muy importante, lo cual evidencia que la educación impartida en las aulas de clase es insuficiente, bien sea debido a que los docentes no se encuentran capacitados, o a que los estudiantes buscan algo más, que responda a iniciativas propias para incrementar su desarrollo intelectual en concordancia con un pensamiento reflexivo y crítico.

Igualmente cabe destacar que aun cuando las matemáticas son un área de difícil aprendizaje, la investigación evidenció un alto nivel de conciencia sobre ella, pues para el $91 \%$ de los estudiantes las matemáticas y las ciencias de la computación están completamente o muy relacionadas, lo cual está en correspondencia con el perfil profesional de la ingeniería de sistemas o afines, en el cual la matemática es un área imprescindible que permite desarrollar un pensamiento lógico necesario en muchos sectores tecnológicos; y de la mano con la ciencia y la tecnología aumentan la probabilidad de éxito laboral y crecimiento económico personal y de la sociedad en general. Esto da a entender que las instituciones educativas deben hacer mayores esfuerzos en promover y garantizar una mejor capacitación de los alumnos en las áreas de matemáticas, aplicando estrategias como las aulas virtuales de matemáticas, tutorías personalizadas, talleres de refuerzo, entre otros, y actividades que incentiven la participación activa de los estudiantes, como olimpiadas y concursos de matemáticas. En cuanto a la enseñanza de la programación, si bien es cierto que los gobiernos han avanzado en la expedición de nuevas leyes educativas y de enseñanza, aún falta mucho por recorrer. Sobre todo en la adecuación de infraestructura tecnológica y física en planteles educativos con bajo presupuesto gubernamental o ubicados en zonas rurales y de difícil acceso.

De acuerdo con los resultados obtenidos, se concluye que el uso del RAE Khan Academy fue muy positivo, pues muestran que para el $83 \%$ de los estudiantes la herramienta logró resolver to- 
talmente o gran parte de sus interrogantes. Así mismo, para el 67 $\%$ los temas tratados en los cursos seleccionados les permitieron aprender o reforzar sus conocimientos, y para el $82 \%$ el uso de la plataforma obtuvo una calificación entre 4 y 5 puntos de aceptación. Lo anterior demuestra que la selección de Khan Academy por parte de los investigadores fue acertada; el hecho de que ofrece acceso completamente gratuito a aprendizaje dinámico y variedad en los temas, convierte la plataforma en un componente eficaz en el proceso de enseñanza-aprendizaje.

Por último, se evidencia la necesidad de reformar los contenidos curriculares, donde la relación entre matemática y computación, pieza fundamental en el desarrollo de nuevas tecnologías, debe ser impartida con enfoque constructivista y conectivista desde los primeros niveles de la educación básica, apoyada en herramientas digitales que permitan al estudiante desarrollar desde muy temprana edad sus capacidades y el interés hacia carreras STEM, y que unido a un cambio de paradigma y capacitación adecuada en los maestros, adapten la educación a las necesidades actuales de la sociedad.

\section{Referencias bibliográficas}

Acharya, U., Oh, S. L., Hagiwara, Y., Tan, J. H. y Adeli, H. (2018). Deep convolutional neural network for the automated detection and diagnosis of seizure using EEG signals. Computers in Biology and Medicine, 100(1), 270-278. DOI: https://doi.org/10.1016/j.compbiomed.2017.09.017

Alshammari, M. y Rezgui, A. (2020). An all pairs shortest path algorithm for dynamic graphs. International Journal of Mathematics and Computer Science, 15(1), 347-365. Recuperado de https://bit.ly/2VBDrob

Alvites, C. (2017). Herramientas TIC en el aprendizaje en el área de matemática: caso Escuela PopUp, Piura-Perú. Hamut'ay, 4(1), 18-30. DOI: http://dx.doi.org/10.21503/hamu.v4i1.1393 
Alzahrani, M. (2017). The effect of using online discussion forums on students' learning. Turkish Online Journal of Educational Technology, 16(1), 164-176. DOI: https://bit.ly/34MhK8Y

Arba, R. (2019). A model of costs simulation using Monte Carlo method for road pavement development projects. Journal of Applied Computer Science \& Mathematics, 13(28). DOI: https://doi.org/10.4316/ JACSM.201902001

Aranda, C. y Callejo, M. (2017). Formas de aproximar el área bajo una curva: un estudio con estudiantes de bachillerato. Enseñanza de las Ciencias, 35(1), 157-174. DOI: http://dx.doi.org/10.5565/rev/ ensciencias. 2075

Ashir, A., Akdemir, B. y Ahmed, B. (2018). Manhattan penalty based multi-modal system for facial recognition. Scholars Journal of Research in Mathematics and Computer Science, 3(1). Recuperado de https://bit. Iy/3cnJJP5

Badmus, N., Alexander, N. y Florence, A. (2020). Statistical analysis of Ebola virus disease: A regression analysis approach. International Journal of Mathematics \& Computation, 31(1). Recuperado de https:// bit.ly/2KaeVFm

Bouroubi, S., Charchali, F. y Benyahia, N. (2019). Set partitions: Solution for sharing secret keys. Romanian Journal of Mathematics and Computer Science, 9(2), 78-86. Recuperado de https://bit.ly/2RJJtSH

Briz, Á. y Serrano, Á. (2018). Aprendizaje de las matemáticas a través del lenguaje de programación $\mathrm{R}$ en educación secundaria. Educación Matemática, 30(1), 133-162. DOI: https://dx.doi.org/10.24844/ em3001.05

Byrd, R. (2018). Cybersecurity: 1) What math is necessary and 2) Developing ubiquitous cybersecurity in current computing programs. Journal of Computing Sciences in Colleges. Recuperado de https://bit. ly/2VeXolC 
Cadena-Iñiguez, P., Rendón, R., Aguilar, J., Salinas, E., Cruz, F. de la y Sangerman, D. (2017). Métodos cuantitativos, métodos cualitativos o su combinación en la investigación: un acercamiento en las ciencias sociales. Revista Mexicana de Ciencias Agrícolas, 8(7), 1603-1617. Recuperado de https://bit.ly/2Vih1Jl

Cárdenas-Guevara, D. (2015). La ingeniería y las ciencias naturales. Revista Facultad de Ingeniería, 24(40), 7-8. Recuperado de https://bit. ly/2XUWSeD

Cariow, A., Cariowa, G. y Majorkowska, D. (2020). An algorithm for Quaternion-Based 3D rotation. International Journal of Applied Mathematics and Computer Science, 30(1), 149-160. DOI: https://doi. org/10.34768/amcs-2020-0012

Colciencias (Departamento Administrativo de Ciencia, Tecnología e Innovación). (2018). Plan Nacional de Ciencia, Tecnología e Innovación para el Desarrollo del Sector de las Tecnologías de la Información y las Comunicaciones (TIC), 2017-2022. Recuperado de https://bit. ly/34H4OkX

Coto, M. (2020). Descubrimiento del estilo de aprendizaje dominante en estudiantes de Matemática Superior. Revista Educación, 44(1). DOI: https://doi.org/10.15517/revedu.v44i1.38571

Cruz, M., Portillo, H., Tchoshanov, M., Flores, S. y Ramírez, O. (2019). Caracterización de las prácticas sociales asociadas a la génesis de la inducción matemática: un estudio exploratorio en estudiantes de ingeniería. Revista Educación, 43(2). DOI: https://doi.org/10.15517/ revedu.v43i2.30493

Díaz, K., Fierro, E. y Muñoz, M. (2018). La enseñanza de la programación: una experiencia en la formación de profesores de Informática. Educación, 27(53), 73-91. DOI: https://dx.doi.org/10.18800/educacion.201802.005

Domínguez, P., Oliveros, M., Coronado, M. y Valdez, B. (2019). Retos de ingeniería: enfoque educativo STEM+A en la revolución industrial 
4.0. Innovación educativa, 19(80), 15-32. Recuperado de https://bit. ly/3bcXEHv

Dove, A., y Dove, E. (2017). Flipping preservice elementary teachers' mathematics anxieties. Contemporary Issues in Technology and Teacher Education, 17(3). Recuperado de https://bit.ly/2XN1piZ

Elaiw, A. y Alshaikh, M. (2020). Stability of a general discrete-time HIV dynamics model with three categories of infected CD4+ T-cells and multiple time delays. JMCS, 20(4), 264-282. DOI: http://dx.doi. org/10.22436/jmcs.020.04.01

Emeda, P., Mwalili, S. y Otieno, G. (2020). Deterministic modeling for HIV and AIDS epidemics with viral load detectability. Journal of Mathematical and Computational Science, 10(4), 728-757. DOI: https:// doi.org/10.28919/jmcs/4474

Fonseca, J. y Alfaro, C. (2018). El cálculo diferencial e integral en una variable en la formación inicial de docentes de matemática en Costa Rica. Revista Educación, 42(2). DOI: https://doi.org/10.15517/revedu. v42i2. 25844

Fuentes, M. y González, J. (2019). Evaluación inicial del diseño de unidades didácticas STEM gamificadas con TIC. Revista Electrónica De Tecnología Educativa, 70, 1-17. DOI: https://doi.org/10.21556/ edutec.2019.70.1469

García, G. y Romero, J. (2018). Matemáticas para todos en tiempos de la inclusión como imperativo: un estudio sobre el programa Todos a Aprender. Revista Colombiana de Educación, 74, 289-310. DOI: https:// doi.org/10.17227/rce.num74-6911

García, M. (2018). Proyecto Newton: Matemáticas para la Vida. Revista Números, 98, 45-58. Recuperado de https://bit.ly/2KaRuvt

García, M., Gómez, A., Solano, N. y Fernández, R. (2020). Las creencias de los futuros maestros sobre la enseñanza y aprendizaje de las 
matemáticas. Revista Espacios, 41(9), 14. Recuperado de https://bit. ly/2 KaOJfH

Gasca, G. y Machuca, L. (2017). Ciencias de la computación en el mundo real. Revista Ibérica de Sistemas e Tecnologias de Informação, 24. DOI: https://dx.doi.org/10.17013/risti.24.0

Ghaleb, F., Taha, A., Hazman, M., Abd ElLatif, M. y Abbass, M. (2020). RDF-BF-Hypergraph representation for relational database. International Journal of Mathematics and Computer Science, 15(1), 41-64. Recuperado de https://bit.ly/2XIzqAZ

Gray, J. y Lindstrøm, C. (2019). Five tips for integrating Khan Academy in your course. The Physics Teacher, 57, 406-408. DOI: https://doi. org/10.1119/1.5124284

Grisales, A. (2018). Uso de recursos TIC en la enseñanza de las matemáticas: retos y perspectivas. Entramado, 14(2), 198-214. DOI: http://dx.doi.org/10.18041/1900-3803/entramado.2.4751

Gürbüz, R. y Erdem, E. (2016). Relationship between mental computation and mathematical reasoning. Cogent Education, 3(1). DOI: https://doi.or g/10.1080/2331186X.2016.1212683

Hasselman, F. y Bosman, A. (2020). Studying complex adaptive systems with internal states: A recurrence network approach to the analysis of multivariate time series data representing self-reports of human experience. Frontiers in Applied Mathematics and Statistics, 6. DOI: https://doi.org/10.3389/fams.2020.00009

Helizar, D., Isnanto, R. y Nurhayati, O. (2018). Analysis knowledge management in implementation enterprise resource planning system using critical success factor (CSF). Scholars Journal of Research in Mathematics and Computer Science, 3(2), 224-237. Recuperado de https://bit.ly/34JSbW5 
Hernández, I. (2018). El Ministerio de Robin Hood: una experiencia de gamificación. Revista Números, 98, 153-162. Recuperado de https://bit. ly/3bfXSgY

Hussein, R., Palangi, H. Ward, R. y Wang, Z. (2019). Optimized deep neural network architecture for robust detection of epileptic seizures using EEG signals. Clinical Neurophysiology, 130(1), 25-37. DOI: https:// doi.org/10.1016/j.clinph.2018.10.010

James, S., Thumbur, G. y Vasudeva, P. (2020). Secure and efficient identity-based proxy signature scheme with message recovery. Journal of Mathematical and Computational Science, 10(3), 448-473. DOI: https://doi.org/10.28919/jmcs/4403

Jara, F., Cancino, P. y Casillas, M. (2019). La integración de KhanAcademy: una estrategia didáctica para la evaluación de matemáticas en ingenierías. Revista Electrónica de Divulgación de Metodologías Emergentes en el Desarrollo de las STEM, 1(1), 26-49. Recuperado de https://bit.ly/3cwvWG4

Jara, I. y Hepp, P. (2016). Enseñar ciencias de la computación: creando oportunidades para los jóvenes de América Latina. Microsoft Corporation. Recuperado de https://bit.ly/2XHQPcZ

Jiménez, A., Garza, A., Méndez, C., Mendoza, J., Acevedo, J., Arredondo, L. y Quiroz, S. (2020). Motivación hacia las matemáticas de estudiantes de bachillerato de modalidad mixta y presencial. Revista Educación, 44(1). DOI: https://doi.org/10.15517/revedu.v44i1.35282

Joshi, H., Sharma, R. y Prajapati, G. (2020). Stability analysis of a deterministic vaccination model with non-monotonic incidence rate. Journal of Mathematical and Computational Science, 10(1), 51-67. DOI: https://doi.org/10.28919/jmcs/4287

Kelly, D. y Rutherford, T. (2017). Khan Academy as supplemental instruction: A controlled study of a computer-based mathematics intervention. International Review of Research in Open and Distributed Learning, 18(4). DOI: https://doi.org/10.19173/irrodl.v18i4.2984 
La República. (28 de agosto de 2019). Computación: millones de programadores se necesitarán en América Latina. La República. Recuperado de https://bit.ly/2xuQfou

Lopez-González, S. (2017). El papel del ingeniero de sistemas en Colombia en la transformación hacia una racionalidad democrática, según la teoría crítica de la tecnología (tesis de maestría). Universidad Eafit, Medellín. Recuperado de https://bit.ly/2VfbLGE

López, V., Couso, D. y Simarro, C. (2020). Educación STEM en y para un mundo digital. Revista de Educación a Distancia, 20(62). DOI: http:// dx.doi.org/10.6018/red.410011

López, V., Mato, D. y Chao, R. (2020). Análisis confirmatorio de la estructura factorial de la ansiedad hacia las matemáticas. Revista de Investigación Educativa, 38(1), 221-237. DOI: http://dx.doi.org/10.6018/ rie.35999

Lluch, L. y Portillo, M. (2018). La competencia de aprender a aprender en el marco de la educación superior. Revista Iberoamericana de Educación, 78(2), 59-76. DOI: https://doi.org/10.35362/rie7823183

Ministerio de Educación y Formación Profesional (MEFP). (2018). Programación, robótica y pensamiento computacional en el aula: situación en España y propuesta normativa. Gobierno de España - MEFP. Recuperado de https://bit.ly/2XKekCc

MEN (Ministerio de Educación Nacional). (2019). Plan Estratégico Institucional 2019-2022. Gobierno de Colombia - MEN. Recuperado de https://bit.ly/2KagMdi

Minte, A., Sepúlveda, A., Díaz, D. y Payahuala, H. (2020). Aprender matemática: dificultades desde la perspectiva de los estudiantes de educación básica y media. Revista Espacios, 41(9), art. 30. Recuperado de https://bit.ly/2RL5r7z

Muhammad, I., Garba, E., Oye, N. y Wajiga, G. (2019). Modeling techniques for knowledge representation of expert system: A survey. 
Journal of Applied Computer Science \& Mathematics, 13(28), 39-44. DOI: https://doi.org/10.4316/JACSM.201902006

OECD. (2016). Education in Colombia. Reviews of National Policies for Education. París: OECD.DOI:https://dx.doi.org/10.1787/9789264250604en

Olarte, J. (2020). Homogeneizar la práctica de la modelación: un reto del sistema educativo colombiano. Revista Educación, 44(1). Recuperado de https://bit.ly/2VFTJfX

Ortiz, E., Santos, J. y Marín, S. (2020). Evaluación continua en la enseñanza universitaria de la contabilidad. Revista de Investigación Educativa, 38(1), 109-129. DOI: http://dx.doi.org/10.6018/rie.329781

Ortiz, L. y Padilla, E. (2017). Taller: Geogebra como herramienta para contribuir con el aprendizaje del cálculo integral en una variable. Artículo presentado en el $V$ Encuentro Enseñanza de la Matemática UNED: Tendencias Actuales en Educación Matemática. Recuperado de https://bit.ly/2Kfg6mQ

Palmas, S. (2018). La tecnología digital como herramienta para la democratización de ideas matemáticas poderosas. Revista Colombiana de Educación, (74), 109-132. DOI: https://doi.org/10.17227/rce.num746900

Popoli, A., Cristofolini, A. y Sandrolini, L. (2020). A numerical model for the calculation of electromagnetic interference from power lines on nonparallel underground pipelines. Mathematics and Computers in Simulation. DOI: https://doi.org/10.1016/j.matcom.2020.02.015

Prieto, G. y Cabrera, D. (2020). Diseño y evaluación de una estrategia lúdica de aprendizaje para enseñar Simulación de Montecarlo. Revista Espacios, 41(13), art. 4. Recuperado de https://bit.ly/2Vhvup7

Ramírez, N., Aparicio, L. y Gómez, E. (2019). Supervised classifiers of prostate cancer. Visión Electrónica, 2(1) Edición Especial. Recuperado de https://bit.ly/2VFd6W7 
Rao, A., Hilton III, J. y Harper, S. (2017). Khan Academy videos in chinese: A case study in OER revision. The International Review of Research in Open and Distributed Learning, 18(5). DOI: https://doi.org/10.19173/ irrodl.v18i5.3086

Reyes, G., Saavedra, J. y Aguayo, M. (2020). Aprendizaje basado en equipos en un curso de Ingeniería en educación superior. Revista Educación, 44(1). DOI: https://doi.org/10.15517/revedu.v44i1.38316

Revelo, J. y Carrillo, S. E. (2018). Impacto del uso de las TIC como herramientas para el aprendizaje de la matemática de los estudiantes de educación media. Revista Cátedra, 1(1), 70-91. Recuperado de https:// bit.ly/2VBS4YC

Romero, H., Cano, L., Charry, C. y Pardo, J. (2019). Deficiencia de adquisición de competencias mínimas en estudiantes de desarrollo de software: hacia un nuevo modelo de enseñanza pedagógico praxeológico. Inclusión y Desarrollo, 1(6). Recuperado de https://bit.ly/2yjt3to

Rubio, I. (24 de septiembre de 2019). Las matriculaciones en carreras técnicas bajan pese a la demanda laboral. El País. Recuperado de https:// bit.ly/2z9rgra

Rueda, K. y Rey, M. (2018). The learning of differential calculus mediated by the platform Khan-Academy. Journal of Physics: Conference Series, 1126. DOI: https://doi.org/10.1088/1742-6596/1126/1/012047

Rumiche, R., Matas, A. y Ríos, J. (2020). Competencias digitales de estudiantes de la Universidad Católica de Santo Toribio de Mogrovejo (Perú). Revista Espacios, 41(9), art. 18. Recuperado de https://bit. Iy/3biy3Nq

Salas, D., Poveda, G., Mesa, O. y Marwan, N. (2020). Generalized synchronization between ENSO and hydrological variables in Colombia: A recurrence quantification approach. Frontiers in Applied Mathematics and Statistics, 6. DOI: https://doi.org/10.3389/fams.2020.00003 
Saouabi, M. y Ezzati, A. (2020). Data mining classification algorithms. International Journal of Mathematics and Computer Science, 15(1), 389394. Recuperado de https://bit.ly/3bjnDNr

Segade, M. y Naya, M. (2018). Secuencia didáctica para el estudio de los triángulos en educación primaria con Geogebra y un primer análisis. Revista Números, 98, 163-177. Recuperado de https://bit.ly/2Kf5vZm

Segarra, J., Pérez, E., Moya, E., Ayuso, P. y Beltrán, H. (2020). Deep learning-based forecasting of aggregated CSP production. Mathematics and Computers in Simulation. DOI: https://doi.org/10.1016/j. matcom.2020.02.007

Shen, J. (2020). An uncertain sustainable supply chain network. Applied Mathematics and Computation, 378. DOI: https://doi.org/10.1016/j. amc. 2020.125213

Solar, H. (2018). Implicaciones de la argumentación en el aula de matemáticas. Revista Colombiana de Educación, 74, 155-176. DOI: https://doi.org/10.17227/rce.num74-6902

Sobalvarro, L. y Camacho, M. (2018). El aprendizaje de la noción de objeto según la forma en niños de educación preescolar: propuesta geometría en movimiento. Revista Educación, 42(2). DOI: https://doi. org/10.15517/revedu.v42i2.28195

Tapia, I. (2019). El poder de Khan Academy en el aprendizaje de las matemáticas en el Conalep. Revista RedCA, 1(3), 120-142. Recuperado de https://bit.ly/2XMPQsb

Tenório, M., Lopes, R., Góis, L. y Junior, G. (2018). Influence of gamification on Khan Academy in Brazilian high school. Pupil: International Journal of Teaching, Education and Learning, 2(2), 51-65. Recuperado de https:// bit.ly/2wRJvkb

Trejo, J., Fajardo, D. y Gutiérrez, J. (2020). A genetic algorithm for the maximum 2-packing set problem. International Journal of Applied 
Mathematics and Computer Science, 30(1), 173-184. DOI: https://doi. org/10.34768/amcs-2020-0014

Türk, O. y Özerdem, M. (2019). Epilepsy detection by using scalogram based convolutional neural network from EEG signals. Brain Sciences, 9(5), 115. DOI: https://doi.org/10.3390/brainsci9050115

Ullah, I., Hussain, M., Qazi, E. y Aboalsamh, H. (2018). An automated system for epilepsy detection using EEG brain signals based on deep learning approach. Expert Systems with Applications, 107(1), 61-71. DOI: https://doi.org/10.1016/j.eswa.2018.04.021

Unesco. (2015). A basic guide to open educational resources (OER). París: Unesco. Recuperado de https://bit.ly/2VeV0eP

Valbuena, S., Muñiz, L. y Berrío, J. (2020). El rol del docente en la argumentación matemática de estudiantes para la resolución de problemas. Revista Espacios, 41(9), art. 15. Recuperado de https://bit. Iy/2VPFdIV

Valbuena, S., Padilla, I. y Rodríguez, E. (2018). El juego y la inteligencia lógico-matemática de estudiantes con capacidades excepcionales. Educación y Humanismo, 20(35), 166-183. DOI: https://doi.org/10.17081/ eduhum.20.35.2964

Vargas, V., Escalante, C. y Carmona, G. (2017). Competencias matemáticas a través de la implementación de actividades provocadoras de modelos. Educación Matemática, 30(1), 213-236. Recuperado de https://bit. ly/2Vie0ZQ

Vesga, G. y Losada, M. de (2018). Creencias epistemológicas de docentes de matemáticas en formación y en ejercicio sobre las matemáticas, su enseñanza y aprendizaje. Revista Colombiana de Educación, 74, 243267. Recuperado de DOI: https://doi.org/10.17227/rce.num74-6909

Vilca, R. (2019). Aplicación del software Geogebra y su influencia en el aprendizaje de áreas y volúmenes de sólidos de revolución en el cálculo integral en los estudiantes del primer año de la Facultad de Ingenierías 
de la Universidad Continental Arequipa - 2017 (tesis de maestría). Universidad Nacional de San Agustín de Arequipa, Perú. Recuperado de https://bit.ly/3ahX5en

Wenqi, W. y Jian, W. (2019). Comparison of multivariate statistical analysis methods. Journal of Mathematical and Computational Science, 9(6), 702-706. DOI: https://doi.org/10.28919/jmcs/4212

Yuan, G., Liu, Y., Huang, W. y Hu, B. (2020). Differentiating grade in breast invasive ductal carcinoma using texture analysis of MRI. Computational and Mathematical Methods in Medicine, 2020. DOI: https://doi. org/10.1155/2020/6913418

Yusuf, I., Lado, A. y Ali, U. (2020). Reliability analysis of communication network system with redundant relay station under partial and complete failure. Journal of Mathematical and Computational Science, 10(4), 863880. DOI: https://doi.org/10.28919/jmcs/4408

Zaldívar, A. (2019). Laboratorios reales versus laboratorios virtuales en las carreras de ciencias de la computación. IE, Revista de investigación educativa de la REDIECH, 10(18), 9-22. DOI: https://dx.doi.org/10.33010/ ie_rie_rediech.v10i18.454

Zengin, Y. (2017). Investigating the use of the Khan Academy and mathematics software with a flipped classroom approach in mathematics teaching. Journal of Educational Technology \& Society, 20(2), 89-100. Recuperado de https://bit.ly/3exofhv

Zhao, W., Wang, W., Jiang, X., Zhang, X., Peng, Y., Zhang, B. y Zhang, G. (2020). A novel deep neural network for robust detection of seizures using EEG signals. Computational and Mathematical Methods in Medicine, 2020. DOI: https://doi.org/10.1155/2020/9689821

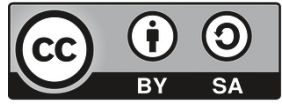

